\title{
On Partial Complete Controllability of Semilinear Systems
}

\author{
Agamirza E. Bashirov and Maher Jneid \\ Eastern Mediterranean University, Gazimagusa, North Cyprus, P.O. Box 95, Mersin 10, Turkey \\ Correspondence should be addressed to Agamirza E. Bashirov; agamirza.bashirov@emu.edu.tr
}

Received 27 March 2013; Revised 28 May 2013; Accepted 11 June 2013

Academic Editor: Sakthivel Rathinasamy

Copyright (C) 2013 A. E. Bashirov and M. Jneid. This is an open access article distributed under the Creative Commons Attribution License, which permits unrestricted use, distribution, and reproduction in any medium, provided the original work is properly cited.

Many control systems can be written as a first-order differential equation if the state space enlarged. Therefore, general conditions on controllability, stated for the first-order differential equations, are too strong for these systems. For such systems partial controllability concepts, which assume the original state space, are more suitable. In this paper, a sufficient condition for the partial complete controllability of semilinear control system is proved. The result is demonstrated through examples.

\section{Introduction}

A concept of controllability, defined by Kalman [1] in 1960 for finite dimensional control systems, is a property of attaining every point in the state space from every initial state point for a finite time. Further studies on this concept in infinite dimensional spaces demonstrated that it is suitable to consider its two versions: a stronger version of complete controllability and a weaker version of approximate controllability. The reason for these versions was the fact that many infinite dimensional control systems are not completely controllable while they are approximately controllable (see Fattorini [2] and Russell [3]). The necessary and sufficient conditions for complete and approximate controllability concepts are almost completely studied and presented in, for example, Curtain and Zwart [4], Bensoussan [5], Bensoussan et al. [6], Zabczyk [7], Bashirov [8], Klamka [9], and so forth for linear systems; Balachandran and Dauer [10, 11], Klamka [12], Mahmudov [13], Li and Yong [14], and so forth for nonlinear systems; Sakthivel et al. [15-17], Yan [18], and so forth for fractional differential systems; and Ren et al. [19] for differential inclusions.

Recently, in Bashirov et al. $[20,21]$ the partial controllability concepts were initiated. The idea of these concepts is that some control systems, including higher order differential equations, wave equations, and delay equations, can be written as a first-order differential equation only by enlarging the dimension of the state space. Therefore, the theorems on controllability, which are formulated for control systems in the form of first-order differential equation, are too strong for them because they involve the enlarged state space. In such cases the partial controllability concepts became preferable, which assume the original state space. The basic controllability conditions for linear systems, including resolvent conditions from Bashirov and Mahmudov [22] and Bashirov and Kerimov [23] (see also [24-26]), are extended to partial controllability concepts by just a replacement of the controllability operator by its partial version.

In this paper our aim is to study the partial complete controllability of semilinear systems. The controllability concepts for semilinear systems are intensively discussed in the literature (see Balachandran and Dauer [10, 11], Klamka [12], Mahmudov [13], Sakthivel et al. [15, 17], and references therein). A basic tool of study in these works is fixed point theorems. In this paper, we also use one of the fixed point theorems, a contraction mapping theorem, and find a sufficient condition for the partial complete controllability of a semilinear control system.

The rest of this paper is organised in the following way. In Section 2 we set the problem, give basic definitions, and motivate the partial controllability concepts by considering a higher order differential equation, a wave equation, and a delay equation. Section 3 contains the proof of the main result. In Section 4, we demonstrate the main result in the examples. Finally, Section 5 contains directions of further research regarding partial controllability concepts. 


\section{Setting the Problem and Motivation}

Consider the basic semilinear control system

$$
x_{t}^{\prime}=A x_{t}+B u_{t}+f\left(t, x_{t}, u_{t}\right), \quad 0<t \leq T, x_{0} \in X,
$$

on the interval $[0, T]$ with $T>0$, where $x$ and $u$ are state and control processes. We assume that the following conditions hold.

(A) $X$ and $U$ are separable Hilbert spaces, $H$ is a closed subspace of $X$, and $L$ is a projection operator from $X$ to $H$;

(B) $A$ is a densely defined closed linear operator on $X$, generating a strongly continuous semigroup $e^{A t}, t \geq$ 0 ;

(C) $B$ is a bounded linear operator from $U$ to $X$;

(D) $f$ is a nonlinear function from $[0, T] \times X \times U$ to $X$, satisfying that

(i) $f$ is continuous on $[0, T] \times X \times U$;

(ii) $f$ is Lipschitz continuous with respect to $x$ and $u$ that is, for all $t \in[0, T], u, v \in U$ and $x, y \in X$,

$\|f(t, x, u)-f(t, y, v)\| \leq K(\|x-y\|+\|u-v\|)$

for some $K \geq 0$;

(E) $U_{\text {ad }}=C(0, T ; U)$ is the space of all continuous functions from $[0, T]$ to $U$.

Define the controllability and $L$-partial controllability operators $Q_{t}$ and $\widetilde{Q}_{t}$ by

$$
Q_{t}=\int_{0}^{t} e^{A s} B B^{*} e^{A^{*} s} d s, \quad \widetilde{Q}_{t}=L Q_{t} L^{*},
$$

where $L^{*}$ is the adjoint of $L$. The $L$-partial controllability operator becomes the controllability operator if $L=I$ (the identity operator). We will also assume the following condition;

(F) $\widetilde{Q}_{T}$ is coercive; that is, there is $\gamma>0$ such that $\left\langle\widetilde{Q}_{T} h, h\right\rangle \geq \gamma\|h\|^{2}$ for all $h \in H$.

Note that this condition implies the existence of $\widetilde{Q}_{T}^{-1}$ as a bounded linear operator and $\left\|\widetilde{Q}_{T}^{-1}\right\| \leq 1 / \gamma$. Respectively, the linear system associated with (1) (the case when $f=0$ ) is $L$-partially complete controllable on the interval $[0, T]$ (see, Bashirov et al. [20, 21]).

The above conditions imply the existence of a unique continuous solution of (1) in the mild sense for every $u \in U_{\mathrm{ad}}$ and $x_{0} \in X$ (see Li and Yong [14] and Byszewski [27]); that is, there is a unique continuous function $x$ from $[0, T]$ to $X$ such that

$$
x_{t}=e^{A t} x_{0}+\int_{0}^{t} e^{A(t-s)}\left(B u_{s}+f\left(s, x_{s}, u_{s}\right)\right) d s
$$

Let

$$
D_{T}^{x_{0}}=\left\{h \in H: \exists u \in U_{\text {ad }} \text { such that } h=L x_{T}\right\} .
$$

Following Bashirov et al. [21], the semilinear control system (1) is said to be $L$-partially complete controllable on $U_{\text {ad }}$ if $D_{T}^{x_{0}}=H$ for all $x_{0} \in X$. Similarly, the semilinear system in (1) is said to be $L$-partially approximate controllable on $U_{\text {ad }}$ if $\overline{D_{T}^{x_{0}}}=H$ for all $x_{0} \in X$, where $\overline{D_{T}^{x_{0}}}$ is the closure of $D_{T}^{x_{0}}$. If $H=X$, these are just well-known complete and approximate controllability concepts, respectively. In this paper, we study the concept of $L$-partial complete controllability.

The reason for studying $L$-partial controllability concepts is that many systems can be written in the form of (1) if the original state space is enlarged. Therefore, suitable controllability concepts for such systems are the $L$-partial controllability concepts with the operator $L$ projecting the enlarged state space to the original one. Here are some examples of such systems, which are discussed in Bashirov [8], Section 3.1.1, in more details.

Example 1. Consider the system

$$
x_{t}^{(n)}=f\left(t, x_{t}, x_{t}^{\prime}, \ldots, x_{t}^{(n-1)}, u_{t}\right),
$$

assuming that its state space is the one-dimensional space $\mathbb{R}$. The ordinary controllability concepts for this system are the equality to or denseness in $\mathbb{R}$ of the respective attainable set. We can write this system as the first-order differential equation

$$
y_{t}^{\prime}=A y_{t}+F\left(t, y_{t}, u_{t}\right)
$$

if

$$
\begin{gathered}
y_{t}=\left[\begin{array}{c}
x_{t} \\
x_{t}^{\prime} \\
\vdots \\
x_{t}^{(n-2)} \\
x_{t}^{(n-1)}
\end{array}\right], \quad A=\left[\begin{array}{ccccc}
0 & 1 & \cdots & 0 & 0 \\
0 & 0 & \cdots & 0 & 0 \\
\vdots & \vdots & \ddots & \vdots & \vdots \\
0 & 0 & \cdots & 0 & 1 \\
0 & 0 & \cdots & 0 & 0
\end{array}\right], \\
F(t, y, u)=\left[\begin{array}{c}
0 \\
0 \\
\vdots \\
0 \\
f\left(t, x, x^{\prime}, \ldots, x^{(n-1)}, u\right)
\end{array}\right] .
\end{gathered}
$$

The state space of this system is the $n$-dimensional Euclidean space $\mathbb{R}^{n}$ and, respectively, its attainable set is a subset of $\mathbb{R}^{n}$. Therefore, the controllability concepts of the system for $y$ are stronger than those of the system for $x$. But if we define the projection operator $L$ by

$$
L=\left[\begin{array}{lllll}
1 & 0 & \cdots & 0 & 0
\end{array}\right]: \mathbb{R}^{n} \longrightarrow \mathbb{R},
$$

then the $L$-partial controllability concepts of the system for $y$ become the same as the ordinary controllability concepts of the system for $x$.

Example 2. Consider the nonlinear wave equation

$$
\frac{\partial^{2} x_{t, \theta}}{\partial t^{2}}=\frac{\partial^{2} x_{t, \theta}}{\partial \theta^{2}}+f\left(t, x_{t, \theta}, \frac{\partial x_{t, \theta}}{\partial t}, u_{t}\right),
$$


where $x$ is a real-valued function of two variables $t \geq 0$ and $0 \leq \theta \leq 1$. The state space of this system is $L_{2}(0,1)$ (the space of square integrable functions on $[0,1])$. This system can be written as the first-order abstract differential equation

$$
y_{t}^{\prime}=A y_{t}+F\left(t, y_{t}, u_{t}\right)
$$

if

$$
\begin{gathered}
y_{t}=\left[\begin{array}{c}
x_{t, \theta} \\
\frac{\partial x_{t, \theta}}{\partial t}
\end{array}\right], \quad A=\left[\begin{array}{cc}
0 & I \\
\frac{d^{2}}{d \theta^{2}} & 0
\end{array}\right], \\
F(t, y, u)=\left[\begin{array}{c}
0 \\
f\left(t, y_{1}, y_{2}, u\right)
\end{array}\right],
\end{gathered}
$$

where $y \in L_{2}(0,1) \times L_{2}(0,1)$. The state space $L_{2}(0,1) \times$ $L_{2}(0,1)$ of the system for $y$ is the enlargement of the state space $L_{2}(0,1)$ of the system for $x$. This is a cost that is paid to bring the wave equation to the form of first-order differential equation. The ordinary controllability concepts for the system (11) are too strong for the system (10). If

$$
L=\left[\begin{array}{ll}
I & 0
\end{array}\right]: L_{2}(0,1) \times L_{2}(0,1) \longrightarrow L_{2}(0,1),
$$

then $L$-partially controllability concepts of the system for $y$ become ordinary controllability concepts of the system for $x$.

Example 3. Consider the system

$$
x_{t}^{\prime}=f\left(t, x_{t}, \int_{-\varepsilon}^{0} x_{t+\theta} d \theta, u_{t}\right),
$$

which contains a simple distributed delay in the nonlinear term, assuming that $x$ is a real-valued function. Then the state space is $\mathbb{R}$. To bring this system to a system without delay, enlarge $\mathbb{R}$ to $\mathbb{R} \times L_{2}(-\varepsilon, 0)$ and define $L_{2}(-\varepsilon, 0)$-valued function

$$
\left[\bar{x}_{t}\right]_{\theta}=x_{t+\theta}, \quad t \geq 0,-\varepsilon \leq \theta \leq 0 .
$$

Then for

$$
\begin{gathered}
y_{t}=\left[\begin{array}{l}
x_{t} \\
\bar{x}_{t}
\end{array}\right], \quad A=\left[\begin{array}{ll}
0 & 0 \\
0 & \frac{d}{d \theta}
\end{array}\right], \\
F(t, y, u)=\left[\begin{array}{c}
f(t, x, \bar{x}, u) \\
0
\end{array}\right],
\end{gathered}
$$

the above system can be written as the abstract system

$$
y_{t}^{\prime}=A y_{t}+f\left(t, y_{t}, u_{t}\right)
$$

Similar to the previous examples, one can easily observe that the ordinary controllability concepts for the system (17) are too strong for the system (14), but the $L$-partial controllability concepts of the system for $y$ with

$$
L=\left[\begin{array}{ll}
I & 0
\end{array}\right]: \mathbb{R} \times L_{2}(0,1) \longrightarrow \mathbb{R}
$$

are exactly the ordinary controllability concepts of the system for $x$.

These examples motivate a study of the partial controllability concepts. In this paper it is proved that under the conditions $(\mathrm{A})-(\mathrm{F})$, the system in (1) is $L$-partially complete controllable.

\section{Main Result}

Denote $\widetilde{X}=C(0, T ; X)$. Then $\widetilde{X} \times U_{\text {ad }}$ is a Banach space with the norm

$$
\|(\cdot, \cdot)\|_{\widetilde{X} \times U_{\mathrm{ad}}}=\|\cdot\|_{\widetilde{X}}+\|\cdot\|_{U_{\mathrm{ad}}} \cdot
$$

Lemma 4. Under the conditions $(A),(B)$, and $(C)$,

$$
\left\|Q_{t}\right\| \leq\left\|Q_{T}\right\|, \quad\left\|\widetilde{Q}_{t}\right\| \leq\left\|\widetilde{Q}_{T}\right\|, \quad 0 \leq t \leq T .
$$

Proof. It is easy to see that $Q_{t}=Q_{t}^{*}$ and $\left\langle Q_{t} x, x\right\rangle \geq 0$ for all $x \in X$. Hence,

$$
\left\|Q_{t}\right\|=\sup _{\|x\|=1}\left\langle Q_{t} x, x\right\rangle .
$$

Then

$$
\begin{aligned}
\left\langle Q_{T} x, x\right\rangle & =\int_{0}^{T}\left\langle e^{A s} B B^{*} e^{A^{*} s} x, x\right\rangle d s \\
& =\left\langle Q_{t} x, x\right\rangle+\int_{t}^{T}\left\langle e^{A s} B B^{*} e^{A^{*} s} x, x\right\rangle d s \\
& =\left\langle Q_{t} x, x\right\rangle+\int_{t}^{T}\left\|B^{*} e^{A^{*} s} x\right\|^{2} d s \\
& \geq\left\langle Q_{t} x, x\right\rangle .
\end{aligned}
$$

This implies $\left\|Q_{t}\right\| \leq\left\|Q_{T}\right\|$. The conclusion of the lemma regarding $\widetilde{Q}_{T}$ follows from $\left\langle\widetilde{Q}_{t} x, x\right\rangle=\left\langle Q_{t} L^{*} x, L^{*} x\right\rangle$.

The proof of the following lemma appears in different forms in several papers, for example, Mahmudov [13]. Our proof is a minor modification of them.

Lemma 5. Assume that the conditions $(A)-(F)$ hold and take arbitrary $h \in H$. Then for the operator $G: \widetilde{X} \times U_{a d} \rightarrow \widetilde{X} \times$ $U_{a d}$, defined by

$$
G(y, v)(t)=(Y(t), V(t)), \quad 0 \leq t \leq T,
$$

where

$$
\begin{aligned}
Y(t)= & -Q_{t} e^{A^{*}(T-t)} L^{*} \widetilde{Q}_{T}^{-1} L \int_{0}^{T} e^{A(T-s)} f\left(s, y_{s}, v_{s}\right) d s \\
& +\int_{0}^{t} e^{A(t-s)} f\left(s, y_{s}, v_{s}\right) d s, \\
V(t)= & B^{*} e^{A^{*}(T-t)} L^{*} \widetilde{Q}_{T}^{-1} \\
& \times\left(h-L e^{A T} x_{0}-L \int_{0}^{T} e^{A(T-s)} f\left(s, y_{s}, v_{s}\right) d s\right),
\end{aligned}
$$

the following inequality holds:

$$
\begin{aligned}
\|G(y, v)-G(z, w)\| \leq & \left(1+\frac{\left\|Q_{T}\right\| M}{\gamma}+\frac{\|B\| M}{\gamma}\right) \\
& \times M K T(\|y-z\|+\|v-w\|),
\end{aligned}
$$

where

$$
M=\sup _{0 \leq t \leq T}\left\|e^{A t}\right\|
$$


Proof. Let $(y, v)$ and $(z, w)$ be two functions in $\widetilde{X} \times U_{\text {ad }}$ such that $G(y, v)=(Y, V)$ and $G(z, w)=(Z, W)$. Then,

$$
\|G(y, v)-G(z, w)\|_{\widetilde{X} \times U_{\mathrm{ad}}}=\|Y-Z\|_{\widetilde{X}}+\|V-W\|_{U_{\mathrm{ad}}} .
$$

Here, $\|Y-Z\|_{\widetilde{X}}$ can be estimated as follows:

$$
\begin{aligned}
\|Y-Z\|=\max _{t \in[0, T]} \| \int_{0}^{t} e^{A(t-s)}\left(f\left(s, y_{s}, v_{s}\right)-f\left(s, z_{s}, w_{s}\right)\right) d s & -Q_{t} e^{A^{*}(T-t)} L^{*} \widetilde{Q}_{T}^{-1} L \\
& \times \int_{0}^{T} e^{A(T-s)}\left(f\left(s, y_{s}, v_{s}\right)-f\left(s, z_{s}, w_{s}\right)\right) d s \| \\
\leq & \max _{t \in[0, T]}\left(M+M^{2}\left\|Q_{t}\right\|\left\|\widetilde{Q}_{T}^{-1}\right\|\right) \\
& \times \int_{0}^{T}\left\|f\left(s, y_{s}, v_{s}\right)-f\left(s, z_{s}, w_{s}\right)\right\| d s \\
\leq & M\left(1+M\left\|Q_{T}\right\|\left\|\widetilde{Q}_{T}^{-1}\right\|\right) \\
& \times \int_{0}^{T}\left\|f\left(s, y_{s}, v_{s}\right)-f\left(s, z_{s}, w_{s}\right)\right\| d s \\
\leq & \left(1+\frac{\left\|Q_{T}\right\| M}{\gamma}\right) M K \int_{0}^{T}\left(\left\|y_{s}-z_{s}\right\|+\left\|v_{s}-w_{s}\right\|\right) d s \\
\leq & \left(1+\frac{\left\|Q_{T}\right\| M}{\gamma}\right) M K T(\|y-z\|+\|v-w\|) .
\end{aligned}
$$

Similarly, for $\|V-W\|_{U_{\text {ad }}}$, we have

$$
\begin{aligned}
\|V-W\|=\max _{t \in[0, T]} \| B^{*} e^{A^{*}(T-t)} L^{*} \widetilde{Q}_{T}^{-1} L \\
\quad \quad \quad \int_{0}^{T} e^{A(T-s)}\left(f\left(s, y_{s}, v_{s}\right)-f\left(s, z_{s}, w_{s}\right)\right) d s \| \\
\leq M^{2}\|B\|\left\|\widetilde{Q}_{T}^{-1}\right\| \int_{0}^{T}\left\|f\left(s, y_{s}, v_{s}\right)-f\left(s, z_{s}, w_{s}\right)\right\| d s \\
\leq M^{2}\|B\| \frac{1}{\gamma} K \int_{0}^{T}\left(\left\|y_{s}-z_{s}\right\|+\left\|v_{s}-w_{s}\right\|\right) d s \\
\leq \frac{\|B\| M}{\gamma} M K T(\|y-z\|+\|v-w\|) .
\end{aligned}
$$

Combining (28) and (29), we obtain the demanded inequality.

Lemma 6. Under the conditions $(A)-(F)$, if

$$
\left(1+\frac{\left\|Q_{T}\right\| M}{\gamma}+\frac{\|B\| M}{\gamma}\right) M K T<1,
$$

then the operator $G$, mapping $\widetilde{X} \times U_{a d}$ into $\widetilde{X} \times U_{a d}$, has a unique fixed point $(x, u) \in \widetilde{X} \times U_{a d}$.
Proof. By Lemma 5, $G$ is a contraction mapping. Also, the space $\widetilde{X} \times U_{\text {ad }}$ is complete. Hence, $G$ has a fixed point.

Theorem 7. Under the conditions $(A)-(F)$ and (30), the semilinear system (1) is L-partially complete controllable on $[0, T]$.

Proof. Take any $x_{0} \in X$ and $h \in H$. Show that there is $u \in U_{\text {ad }}$ such that $h=L x_{T}$. To this end, consider $u$, defined as follows:

$$
\begin{aligned}
u_{t}= & B^{*} e^{A^{*}(T-t)} L^{*} \widetilde{Q}_{T}^{-1} \\
& \times\left(h-L e^{A T} x_{0}-L \int_{0}^{T} e^{A(T-s)} f\left(s, x_{s}, u_{s}\right) d s\right) .
\end{aligned}
$$

Substituting (31) in (4) and applying Fubini's theorem (see Bashirov [8], p. 45), we obtain

$$
\begin{aligned}
x_{t}= & e^{A t} x_{0} \\
& +\int_{0}^{t} e^{A(t-s)} B B^{*} e^{A^{*}(t-s)} e^{A^{*}(T-t)} L^{*} \widetilde{Q}_{T}^{-1}\left(h-L e^{A T} x_{0}\right) d s \\
& -\int_{0}^{t} \int_{0}^{T} e^{A(t-s)} B B^{*} e^{A^{*}(t-s)} e^{A^{*}(T-t)} L^{*} \widetilde{Q}_{T}^{-1} L e^{A(T-r)} \\
& \times f\left(r, x_{r}, u_{r}\right) d r d s \\
& +\int_{0}^{t} e^{A(t-s)} f\left(s, x_{s}, u_{s}\right) d s \\
= & e^{A t} x_{0}+Q_{t} e^{A^{*}(T-t)} L^{*} \widetilde{Q}_{T}^{-1}\left(h-L e^{A T} x_{0}\right) \\
& +\int_{0}^{t} e^{A(t-s)} f\left(s, x_{s}, u_{s}\right) d s \\
& -\int_{0}^{T} \int_{0}^{t} e^{A(t-s)} B B^{*} e^{A^{*}(t-s)} e^{A^{*}(T-t)} L^{*} \widetilde{Q}_{T}^{-1} L e^{A(T-r)} \\
& \times f\left(r, x_{r}, u_{r}\right) d s d r
\end{aligned}
$$




$$
\begin{aligned}
= & e^{A t} x_{0}+Q_{t} e^{A^{*}(T-t)} L^{*} \widetilde{Q}_{T}^{-1}\left(h-L e^{A T} x_{0}\right) \\
& +\int_{0}^{t} e^{A(t-s)} f\left(s, x_{s}, u_{s}\right) d s \\
& -\int_{0}^{T} Q_{t} e^{A^{*}(T-t)} L^{*} \widetilde{Q}_{T}^{-1} L e^{A(T-r)} f\left(r, x_{r}, u_{r}\right) d r \\
= & e^{A t} x_{0}+Q_{t} e^{A^{*}(T-t)} L^{*} \widetilde{Q}_{T}^{-1}\left(h-L e^{A T} x_{0}\right) \\
& +\int_{0}^{t} e^{A(t-s)} f\left(s, x_{s}, u_{s}\right) d s \\
& -Q_{t} e^{A^{*}(T-t)} L^{*} \widetilde{Q}_{T}^{-1} L \int_{0}^{T} e^{A(T-s)} f\left(s, x_{s}, u_{s}\right) d s .
\end{aligned}
$$

According to Lemma 6 , there is a unique pair $(x, u) \in \widetilde{X} \times$ $U_{\text {ad }}$, satisfying (31) and (32). So, $u \in U_{\text {ad }}$. Furthermore, we have

$$
\begin{aligned}
L x_{T}= & L\left(e^{A T} x_{0}+Q_{T} L^{*} \widetilde{Q}_{T}^{-1}\left(h-L e^{A T} x_{0}\right)\right. \\
& +\int_{0}^{T} e^{A(T-s)} f\left(s, x_{s}, u_{s}\right) d s \\
& \left.-Q_{T} L^{*} \widetilde{Q}_{T}^{-1} L \int_{0}^{T} e^{A(T-s)} f\left(s, x_{s}, u_{s}\right) d s\right) \\
= & L e^{A T} x_{0}+L Q_{T} L^{*} \widetilde{Q}_{T}^{-1}\left(h-L e^{A T} x_{0}\right) \\
& +L \int_{0}^{T} e^{A(T-s)} f\left(s, x_{s}, u_{s}\right) d s \\
& -L Q_{T} L^{*} \widetilde{Q}_{T}^{-1} L \int_{0}^{T} e^{A(T-s)} f\left(s, x_{s}, u_{s}\right) d s=h .
\end{aligned}
$$

Thus, there is $u \in U_{\text {ad }}$ which steers $x_{0}$ to $x_{T}$ with $L x_{T}=h$. This means that the semilinear system (1) is $L$-partially complete controllable on $[0, T]$ as desired.

Remark 8. Decomposing $Q_{T}$ in the form

$$
Q_{T}=\left[\begin{array}{ll}
\widetilde{Q}_{T} & R_{T} \\
R_{R}^{*} & P_{T}
\end{array}\right],
$$

where $R_{T}: H^{\perp} \rightarrow H$ and $P_{T}: H^{\perp} \rightarrow H^{\perp}$ are other components of $Q_{T}$ besides $\widetilde{Q}_{T}$ and $H^{\perp}$ is an orthogonal complement of $H$ in $X$, one can calculate

$$
\left\langle Q_{T} h, h\right\rangle=\left\langle\widetilde{Q}_{T} h_{1}, h_{1}\right\rangle+2\left\langle R_{T} h_{2}, h_{1}\right\rangle+\left\langle P_{T} h_{2}, h_{2}\right\rangle
$$

where $h_{1}=L h \in H$ and $h_{2}=h-L h \in H^{\perp}$. Therefore, the coercivity of $Q_{T}$ implies the same of $\widetilde{Q}_{T}$. But, the converse is not true. Theorem 7 is powerful in the cases when $\widetilde{Q}_{T}$ is coercive, but $P_{T}$ is not.

Example 9. Theorem 7 establishes just sufficient condition of $L$-partial complete controllability. In this example we will demonstrate that this is not a necessary condition. We will consider a simple case of $L=I$ when $L$-partial complete controllability reduces to complete controllability. Consider the one-dimensional control system

$$
x_{t}^{\prime}=2 x_{t}+2 u_{t}, \quad x_{0} \in \mathbb{R} .
$$

This is a linear system, and the controllability operator of this system is equal to

$$
\int_{0}^{T} 4 e^{4 t} d t=e^{4 T}-1>0 \quad \text { for every } T>0 .
$$

According to the theory of controllability for linear systems, this system is controllable (completely) for every $T>0$.

Have another look at this system by writing it as

$$
x_{t}^{\prime}=x_{t}+u_{t}+f\left(x_{t}, u_{t}\right), \quad x_{0} \in \mathbb{R},
$$

where

$$
f(x, u)=x+u \text {. }
$$

Here, $f$ satisfies the Lipschitz condition with $K=1$. Also, $A=B=1$, implying $\|B\|=1$ and $M=\sup _{[0, T]}\left\|e^{A t}\right\|=e^{T}$. Furthermore,

$$
Q_{T}=\int_{0}^{T} e^{2 t} d t=\frac{e^{2 T}-1}{2}
$$

So, $\left\|Q_{T}\right\|=\gamma=\left(e^{2 T}-1\right) / 2$. Then the inequality (30) becomes

$$
\left(1+e^{T}+\frac{2 e^{T}}{e^{T}-1}\right) e^{T} T<1 .
$$

The limit of the left-hand side in this inequality when $T \rightarrow$ $\infty$ is equal to $\infty$. This means that there is a sufficiently large $T$ such that the conditions of Theorem 7 do not hold for this $T$, although the system under consideration is completely controllable. Thus, Theorem 7 states a sufficient condition which is not a necessary condition.

\section{Examples}

We demonstrate the features of $L$-partial complete controllability in the following examples of control systems.

Example 1. Consider the system of differential equations

$$
\begin{gathered}
x_{t}^{\prime}=y_{t}+b u_{t}, \quad x_{0} \in \mathbb{R}, \\
y_{t}^{\prime}=f\left(t, x_{t}, y_{t}, u_{t}\right), \quad y_{0} \in \mathbb{R}
\end{gathered}
$$

on $[0, T]$, where $u \in U_{\mathrm{ad}}=C(0, T ; \mathbb{R})$. Besides the complete controllability property, that is,

$$
\left\{(x, y) \in \mathbb{R}^{2}: \exists u \in U_{\text {ad }} \text { such that }\left(x_{T}, y_{T}\right)=(x, y)\right\}=\mathbb{R}^{2},
$$

we can investigate the partial complete controllability property, that is,

$$
\left\{x \in \mathbb{R}: \exists u \in U_{\text {ad }} \text { such that } x_{T}=x\right\}=\mathbb{R} .
$$


We can write this system in $\mathbb{R}^{2}$ as the following semilinear system:

$$
z_{t}^{\prime}=A z_{t}+F\left(t, z_{t}, u_{t}\right)+B u_{t}
$$

where

$$
\begin{gathered}
z_{t}=\left[\begin{array}{l}
x_{t} \\
y_{t}
\end{array}\right], \quad A=\left[\begin{array}{ll}
0 & 1 \\
0 & 0
\end{array}\right] \quad B=\left[\begin{array}{l}
b \\
0
\end{array}\right], \\
F(t, z, u)=\left[\begin{array}{c}
0 \\
f(t, x, y, u)
\end{array}\right],
\end{gathered}
$$

assuming that

$$
z=\left[\begin{array}{l}
x \\
y
\end{array}\right]
$$

It can be calculated that

$$
e^{A t}=\left[\begin{array}{ll}
1 & t \\
0 & 1
\end{array}\right]=\left[\begin{array}{ll}
1 & 0 \\
0 & 1
\end{array}\right]+\left[\begin{array}{ll}
0 & t \\
0 & 0
\end{array}\right] .
$$

Hence,

$$
\left\|e^{A t}\right\| \leq 1+t \leq 1+T, \quad 0 \leq t \leq T .
$$

The controllability operator is

$$
Q_{T}=\int_{0}^{T} e^{A t} B B^{*} e^{A^{*} t} d t=b^{2} T\left[\begin{array}{ll}
1 & 0 \\
0 & 0
\end{array}\right]
$$

Hence, $Q_{T}$ is not coercive, and the conditions for complete controllability, based on coercivity of $Q_{T}$, fail for this example. Although system (42) can still be complete controllable for properly selected functions $f$, we can investigate the partial complete controllability for this system being interested in just the first component $x_{t}$ of $z_{t}$.

Let $L=\left[\begin{array}{ll}1 & 0\end{array}\right]$. Then

$$
\widetilde{Q}_{T}=L Q_{T} L^{*}=b^{2} T>0 .
$$

This means that the linear system associated with the semilinear system (45) is $L$-partially complete controllable. Furthermore, the inequality (30) becomes

$$
\left(1+\frac{b^{2} T(1+T)}{b^{2} T}+\frac{b(1+T)}{b^{2} T}\right)(1+T) T K<1,
$$

or, simplifying,

$$
K<\frac{b}{(1+T)\left(1+T+2 b T+b T^{2}\right)} .
$$

This establishes a relation between Lipschitz coefficient $K$ and terminal time moment $T$. Depending on $K, T$ must be taken sufficiently large to satisfy (53). So, the system (42) is $L$ partially complete controllable for the time $T$ if the Lipschitz coefficient $K$, related to $f$, satisfies (53).
Example 2. Delay equations are typical for application of partial controllability concepts. Consider a nonlinear delay equation

$$
\begin{gathered}
x_{t}^{\prime}=a x_{t}+b u_{t}+f\left(t, x_{t}, \int_{-\varepsilon}^{0} x_{t+\theta} d \theta, u_{t}\right), \\
x_{0}=\xi, \quad x_{\theta}=\eta_{\theta}, \quad-\varepsilon \leq \theta \leq 0,
\end{gathered}
$$

on $[0, T]$, where $0<\varepsilon<T, \eta \in L_{2}(-\varepsilon, 0 ; \mathbb{R})$, and $u \in U_{\text {ad }}=$ $C(0, T ; \mathbb{R})$.

Similar to Example 3, introduce the function $\bar{x}:[0, T] \rightarrow$ $L_{2}(-\varepsilon, 0 ; \mathbb{R})$ by

$$
\left[\bar{x}_{t}\right]_{\theta}=x_{t+\theta}, \quad 0 \leq t \leq T,-\varepsilon \leq \theta \leq 0 .
$$

This function satisfies

$$
\bar{x}_{t}^{\prime}=\left(\frac{d}{d \theta}\right) \bar{x}_{t}, \quad \bar{x}_{0}=\eta, \quad 0<t \leq T .
$$

Denote by $\mathscr{T}_{t}, t \geq 0$, the semigroup generated by the differential operator $d / d \theta$ and let $\Gamma$ be the integral operator from $L_{2}(-\varepsilon, 0 ; \mathbb{R})$ to $\mathbb{R}$, defined by

$$
\Gamma h=\int_{-\varepsilon}^{0} h_{\theta} d \theta, \quad h \in L_{2}(-\varepsilon, 0 ; \mathbb{R}),
$$

noticing that $\|\Gamma\| \leq \sqrt{\varepsilon}$. Then for

$$
y_{t}=\left[\begin{array}{l}
x_{t} \\
\bar{x}_{t}
\end{array}\right], \quad \zeta=\left[\begin{array}{l}
\xi \\
\eta
\end{array}\right] \in \mathbb{R} \times L_{2}(-\varepsilon, 0 ; \mathbb{R}),
$$

we can write system (54) as

$$
y_{t}^{\prime}=A y_{t}+F\left(t, y_{t}, u_{t}\right)+B u_{t}, \quad y_{0}=\zeta,
$$

where

$$
\begin{array}{cc}
A=\left[\begin{array}{cc}
a & 0 \\
0 & \frac{d}{d \theta}
\end{array}\right], \quad & F(t, y, u)=\left[\begin{array}{c}
f(t, x, \Gamma \bar{x}, u) \\
0
\end{array}\right], \\
B=\left[\begin{array}{l}
b \\
0
\end{array}\right],
\end{array}
$$

assuming that

$$
y=\left[\begin{array}{l}
x \\
x
\end{array}\right] \in \mathbb{R} \times L_{2}(-\varepsilon, 0 ; \mathbb{R}) .
$$

The semigroup, generated by $A$, has the form

$$
e^{A t}=\left[\begin{array}{cc}
e^{a t} & 0 \\
0 & \mathscr{T}_{t}
\end{array}\right], \quad t \geq 0 .
$$

Therefore, the controllability operator for system (54) can be calculated as

$$
\begin{aligned}
Q_{T} & =\int_{0}^{T} e^{A t} B^{*} B e^{A^{*} t} d t=\int_{0}^{T}\left[\begin{array}{cc}
b^{2} e^{2 a t} & 0 \\
0 & 0
\end{array}\right] d t \\
& =\left[\begin{array}{cc}
\frac{b^{2}\left(e^{2 a T}-1\right)}{2 a} & 0 \\
0 & 0
\end{array}\right] .
\end{aligned}
$$

This is definitely not a coercive operator. 
Taking into account that the original system is given by (54) and (59) is just representation of (54) in the standard form, which enlarges the original state space $\mathbb{R}$ to $\mathbb{R} \times$ $L_{2}(-\varepsilon, 0 ; \mathbb{R})$, we observe that the complete controllability for system (54) is in fact $L$-partial complete controllability for system (59) if

$$
L=\left[\begin{array}{ll}
1 & 0
\end{array}\right]: \mathbb{R} \times L_{2}(-\varepsilon, 0 ; \mathbb{R}) \longrightarrow \mathbb{R}
$$

Calculating partial controllability operator, we obtain

$$
\widetilde{Q}_{T}=L Q_{T} L^{*}=\frac{b^{2}\left(e^{2 a T}-1\right)}{2 a}>0,
$$

which is coercive.

Furthermore, using

$$
\left\|e^{A t}\right\| \leq 1+e^{a T}, \quad\left\|Q_{T}\right\|=\gamma=\frac{b^{2}\left(e^{2 a T}-1\right)}{2 a},
$$

we write the inequality (30) in the form

$$
\left(1+1+e^{a T}+\frac{2 a}{b\left(e^{a T}-1\right)}\right)\left(1+e^{a T}\right) T K<1 .
$$

If the Lipschitz coefficient $K$ of the function $F$ and terminal time moment $T$ satisfy this inequality, then system (54) is completely controllable, which in turn means that system (59) is $L$-partially complete controllable.

\section{Conclusion}

In this paper a sufficient condition for partial complete controllability of a semilinear control system is proved. This is a continuation of the pioneering research that has been done by Bashirov et al. [20, 21] about partial controllability concepts. A research in this way, concerning partial complete and approximate controllability for semilinear deterministic and stochastic systems, has already been done and awaiting for publication. There are other kinds of systems which besides semilinearity include other features, for example, impulsiveness, fractional derivative issue, and so forth. The result of this paper can be extended to these systems as well.

\section{References}

[1] R. E. Kalman, "A new approach to linear filtering and prediction problems," Journal of Basic Engineering D, Transactions of ASME, vol. 82, pp. 35-45, 1960.

[2] H. O. Fattorini, "Some remarks on complete controllability," SIAM Journal on Control, vol. 4, pp. 686-694, 1966.

[3] D. L. Russell, "Nonharmonic Fourier series in the control theory of distributed parameter systems," Journal of Mathematical Analysis and Applications, vol. 18, pp. 542-560, 1967.

[4] R. F. Curtain and H. J. Zwart, An Introduction to Infinite Dimensional Linear Systems Theory, Springer, Berlin, Germany, 1995.

[5] A. Bensoussan, Stochastic Control of Partially Observable Systems, Cambridge University Press, Cambridge, UK, 1992.
[6] A. Bensoussan, G. Da Prato, M. C. Delfour, and S. K. Mitter, Representation and Control of Infinite Dimensional Systems, Systems \& Control: Foundations \& Applications, Birkhäuser, Boston, Mass, USA, 2nd edition, 2007.

[7] J. Zabczyk, Mathematical Control Theory: An Introduction, Systems \& Control: Foundations \& Applications, Birkhäuser, Boston, Mass, USA, 1995.

[8] A. E. Bashirov, Partially Observable Linear Systems under Dependent Noises, Systems \& Control: Foundations \& Applications, Birkhäuser, Basel, Switzerland, 2003.

[9] J. Klamka, Controllability of Dynamical Systems, vol. 48 of Mathematics and Its Applications, Kluwer Academic Publishers, Dordrecht, The Netherlands, 1991.

[10] K. Balachandran and J. P. Dauer, "Controllability of nonlinear systems in Banach spaces: a survey," Journal of Optimization Theory and Applications, vol. 115, no. 1, pp. 7-28, 2002.

[11] K. Balachandran and J. P. Dauer, "Local controllability of semilinear evolution systems in Banach spaces," Indian Journal of Pure and Applied Mathematics, vol. 29, no. 3, pp. 311-320, 1998.

[12] J. Klamka, "Schauder's fixed-point theorem in nonlinear controllability problems," Control and Cybernetics, vol. 29, no. 1, pp. 153-165, 2000.

[13] N. I. Mahmudov, "Controllability of semilinear stochastic systems in Hilbert spaces," Journal of Mathematical Analysis and Applications, vol. 288, no. 1, pp. 197-211, 2003.

[14] X. J. Li and J. M. Yong, Optimal Control Theory for InfiniteDimensional Systems, Systems \& Control: Foundations \& Applications, Birkhäuser, Boston, Mass, USA, 1995.

[15] R. Sakthivel, N. I. Mahmudov, and J. J. Nieto, "Controllability for a class of fractional-order neutral evolution control systems," Applied Mathematics and Computation, vol. 218, no. 20, pp. 10334-10340, 2012.

[16] R. Sakthivel, R. Ganesh, and S. Suganya, "Approximate controllability of fractional neutral stochastic system with infinite delay," Reports on Mathematical Physics, vol. 70, no. 3, pp. 291311, 2012.

[17] R. Sakthivel, Y. Ren, and N. I. Mahmudov, "On the approximate controllability of semilinear fractional differential systems," Computers \& Mathematics with Applications, vol. 62, no. 3, pp. 1451-1459, 2011.

[18] Z. Yan, "Approximate controllability of partial neutral functional differential systems of fractional order with statedependent delay," International Journal of Control, vol. 85, no. 8, pp. 1051-1062, 2012.

[19] Y. Ren, L. Hu, and R. Sakthivel, "Controllability of impulsive neutral stochastic functional differential inclusions with infinite delay," Journal of Computational and Applied Mathematics, vol. 235, no. 8, pp. 2603-2614, 2011.

[20] A. E. Bashirov, H. Etikan, and N. Şemi, "Partial controllability of stochastic linear systems," International Journal of Control, vol. 83, no. 12, pp. 2564-2572, 2010.

[21] A. E. Bashirov, N. Mahmudov, N. Şemi, and H. Etikan, "Partial controllability concepts," International Journal of Control, vol. 80, no. 1, pp. 1-7, 2007.

[22] A. E. Bashirov and N. I. Mahmudov, "On concepts of controllability for deterministic and stochastic systems," SIAM Journal on Control and Optimization, vol. 37, no. 6, pp. 1808-1821, 1999.

[23] A. E. Bashirov and K. R. Kerimov, "On controllability conception for stochastic systems," SIAM Journal on Control and Optimization, vol. 35, no. 2, pp. 384-398, 1997. 
[24] A. E. Bashirov, "On weakening of the controllability concepts," in Proceedings of the 35th Conference on Decission and Control, pp. 640-645, Kobe, Japan, 1996.

[25] A. E. Bashirov and N. I. Mahmudov, "Controllability of linear deterministic and stochastic systems," in Proceedings of the 38th Conference on Decission and Control, pp. 3196-3201, Phoenix, Aris, USA, 1999.

[26] A. E. Bashirov and N. I. Mahmudov, "Some new results in theory of controllability," in Proceedings of the 7th Mediterranean Conference on Control and Automation, pp. 323-343, Haifa, Israel, 1999.

[27] L. Byszewski, "Theorems about the existence and uniqueness of solutions of a semilinear evolution nonlocal Cauchy problem," Journal of Mathematical Analysis and Applications, vol. 162, no. 2, pp. 494-505, 1991. 


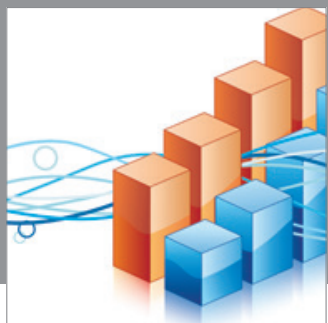

Advances in

Operations Research

mansans

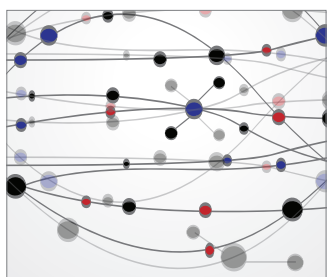

The Scientific World Journal
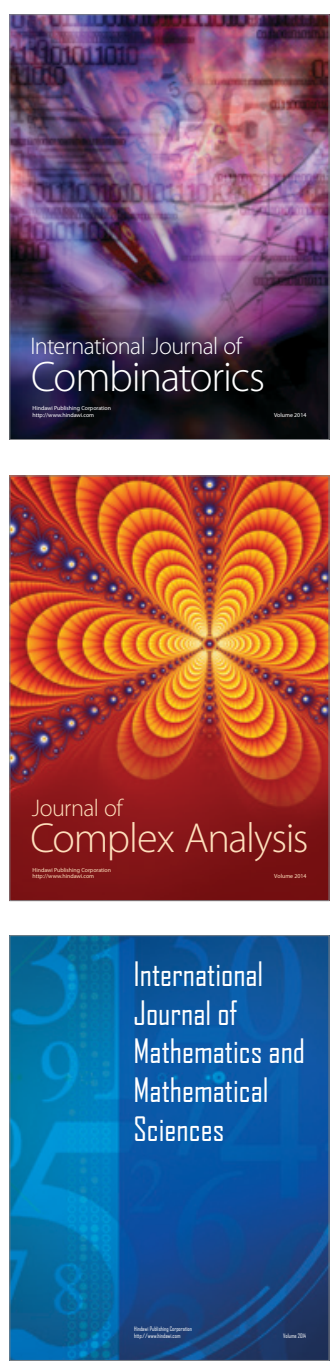
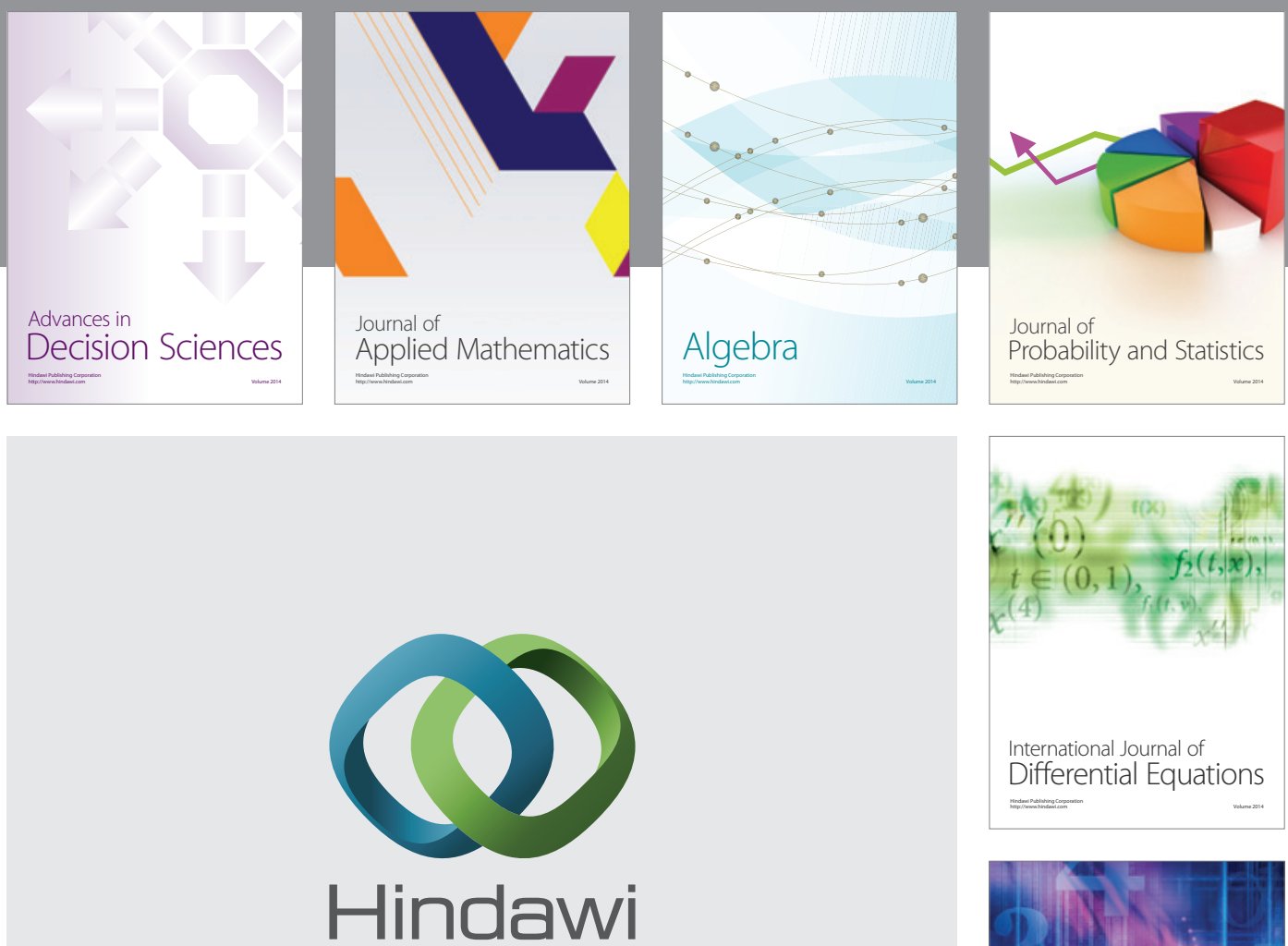

Submit your manuscripts at http://www.hindawi.com
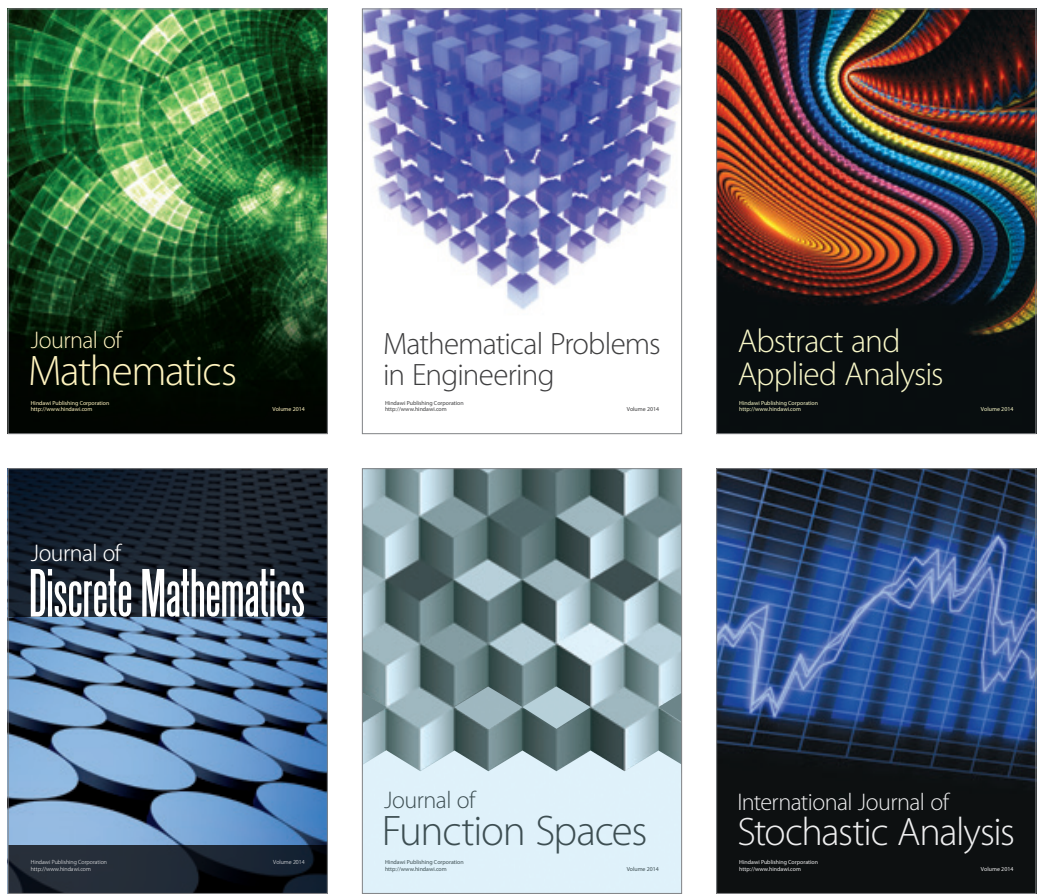

Journal of

Function Spaces

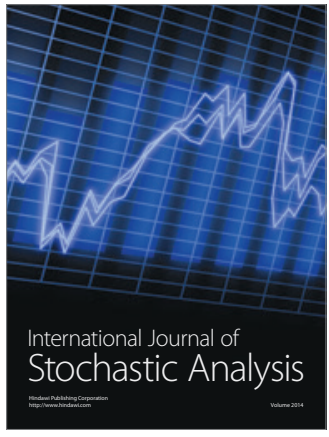

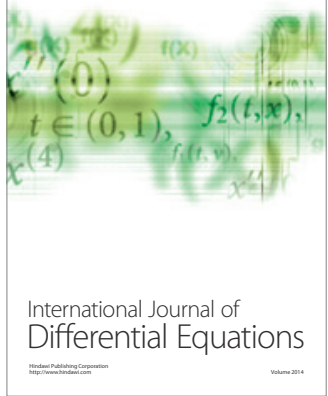
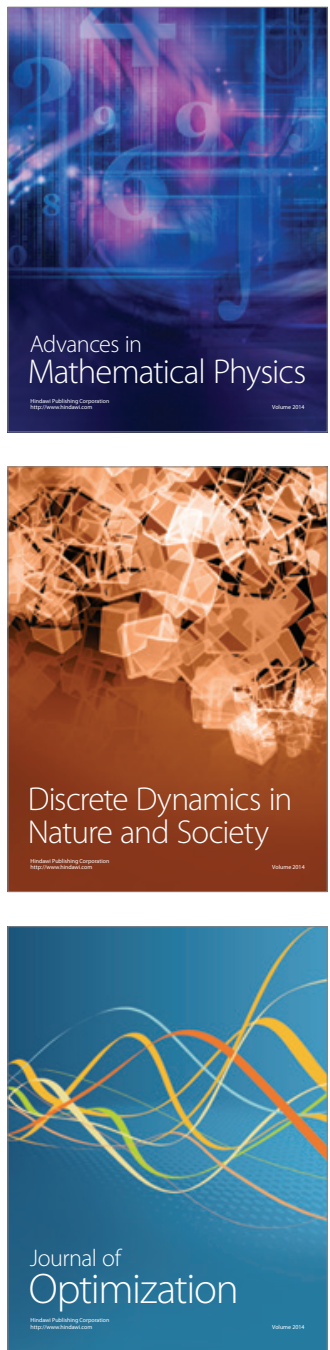